\title{
Array of Sensors: A Spatiotemporal-State-Space Model for Target Trajectory Tracking
}

\author{
A. Manikas, V. Sridhar and Y. I. Kamil \\ (Invited Paper)
}

\begin{abstract}
In this paper, with the objective of tracking the trajectory of multiple mobile targets, a novel spatiotemporal-state-space model is introduced for an array of sensors distributed in space. Under the wideband assumption, the proposed model incorporates the array geometry in conjunction with crucial target parameters namely (i) ranges, (ii) directions, (iii) velocities and (iv) associated Doppler effects. Computer simulation studies show some representative examples where the proposed model is utilised to track the locations of sources in space with a very high accuracy.
\end{abstract}

Index Terms-Array Processing, Spatiotemporal, Tracking, Spherical Wave Propagation

$\begin{array}{ll}A, a & \text { Scalar } \\ \underline{A}, \underline{a} & \text { Column vector } \\ \mathbb{A}, \mathbf{A} & \text { Matrix } \\ (\cdot)^{T},(\cdot)^{H} & \text { Transpose, Hermitian transpose } \\ \odot, \otimes & \text { Hadamard, Kronecker products } \\ \exp (\underline{A}) & \text { Element by element exponential of } \underline{A} \\ \underline{A}^{b} & \text { Element by element power of the vector } \underline{A} \\ \mathcal{E}\{\cdot\} & \text { Expectation operator } \\ \text { vec }(\mathbb{A}) & \text { Vectorization of } \mathbb{A} \text { by stacking its columns } \\ \mathbb{I}_{N} & \text { Identity matrix of dimensions } N \times N \\ \underline{1}_{N} & \text { Column vector of } N \text { ones } \\ \mathcal{R}, \mathcal{C} & \text { Set of real and complex numbers }\end{array}$

\section{INTRODUCTION}

Multiple source/target tracking is an important element of any surveillance and monitoring system. For example, tracking the trajectories of multiple sources can assist in resource management in space [1], air traffic control [2], missile defence [3], asset tracking [4], mobile communications [5] and wireless sensor networks [6][7]. A straightforward approach to solve the problem of tracking multiple sources is to divide the tracking period into sufficiently small time intervals, over which sources may be assumed stationary. In this case, the tracking problem can be solved as a repetitive localisation problem. However, these algorithms begin to exhibit poor performance in the presence of fast moving sources, when the source stationarity over the observation interval is no longer true [8].

An alternative approach to tracking in the literature, is to introduce a state space model to represent target motion dynamics. Using the state space model, various Bayesian approaches may be employed to estimate the locations of targets on a snapshot by snapshot basis [9][10]. These algorithms provide a better performance at the cost of an increased computational complexity. In this paper, in the framework of a state space model, a solution to track multiple mobile sources is presented using a large aperture array of passive sensors with known array geometry connected with fibre, wireline or wireless links. Note that this is distinctly different from the active tracking ([11], [12], [13], [14]) or active localisation (e.g. [15],[16]) approaches discussed extensively for example in the "radar" literature. The approach proposed in this paper differs from the aforementioned approaches on many counts and is based on the utilisation of a fully parametric target environment rather than a statistical modelling. In this paper, due to the large array aperture, the sources are assumed to lie in the near-far field of the array and hence a spherical wave propagation model is employed. Using the spherical wave manifold vector, encompassing the target's parameters and Doppler effects, a modelling based on a spherical wave spatiotemporal manifold vector is developed under the "wideband ${ }^{1}$-assumption". This modelling, in conjunction with the kinematics of the target, enables the formation of a state space model that can be used to track the range and direction of the targets.

The remainder of this paper is organized as follows: In Section II, the received signal vector is modelled under the wideband-assumption for a large aperture of passive sensors using the concept of the spherical spatiotemporal manifold vector. In Section III, the kinematic model of the targets is developed. Using the models in Sections II and III, in Section IV a novel spatiotemporal-state space model for the multi-source tracking problem is presented. Following this, in Section $\mathrm{V}$, some examples of computer simulation results are presented and the paper is concluded in Section VI.

\section{WIDEBAND-ASSUMPTION AND SPATIOTEMPORAL ARRAY MANIFOLD VECTOR}

Consider a fully calibrated large aperture sparse array of $N$ passive sensors with locations in Cartesian coordinates given by the matrix $\mathbf{r} \in \mathcal{R}^{3 \times N}$ with respect to the system origin. That is

$$
\mathbf{r}=\left[\underline{r}_{1}, \underline{r}_{2}, \ldots, \underline{r}_{N}\right]=\left[\underline{r}_{x}, \underline{r}_{y}, \underline{r}_{z}\right]^{T},
$$

where $\underline{r}_{i} \in \mathcal{R}^{3 \times 1}$ for $i=1,2, \cdots, N$ denotes the location of the $i$-th element in the array and $\underline{r}_{x}, \underline{r}_{y}, \underline{r}_{z} \in \mathcal{R}^{N \times 1}$ denote the vectors describing the $x, y$ and $z$ coordinates of the array elements. Without loss of generality, assume the first array sensor is located at the array reference point (i.e., $\underline{r}_{1}=[0,0,0]^{T}$ ). Consider that the array operates in the presence of $M$ targets with common carrier frequency $F_{c}$ where the $i$-th source transmits an unknown message signal $m_{i}(t)$. In addition, it is assumed that the targets move at a constant speed over a given observation interval subject to minor Brownian disturbances [9]

\footnotetext{
${ }^{1}$ If the transmitted wavefront changes when traversing through the sensors of the array (i.e. different sensors see different parts of the transmitted signal) this is defined as the "wideband-assumption" (this should not be confused with the term "wideband signals"). If the array elements are distributed in space with large inter-sensor spacings, this assumption is essential.
} 
acting on their heading directions. Finally, it is assumed that the array aperture $^{2}$ is sufficiently large such that the targets lie in the near-far field of the array and thus undergo spherical wave propagation.

Consider that the large aperture array is tracking the $i$-th target. The baseband signal vector $\underline{x}_{i}(t) \in C^{N \times 1}$ at point $\mathrm{D}$ received by the passive array of sensors at point $\mathrm{C}$ can be modelled, under the wideband-assumption, as

$$
\underline{x}_{i}(t)=\underline{S}_{i}(t) \odot \underline{F}_{i}(t) \odot \underline{\mathrm{m}}_{i}(t)+\underline{n}(t),
$$

where the vector $\underline{S}_{i}(t) \triangleq \underline{S}\left(\theta_{i}(t), \rho_{i}(t)\right) \in \mathcal{C}^{N \times 1}$ represents the $N$-dimensional array manifold (array response) vector, $\underline{F}_{i}(t) \in$ $\mathcal{C}^{N \times 1}$ encompasses the effect of Doppler shift due to the velocity of the target, the vector $\underline{\mathrm{m}}_{i}(t) \in \mathcal{C}^{N \times 1}$ denotes the various delayed versions of the $i$-th target's signal received at the array elements (wideband-assumption) and $\underline{n}(t) \in \mathcal{C}^{N \times 1}$ models the noise. In this paper, $\underline{n}(t) \in \mathcal{C}^{N \times 1}$ is assumed to be zero mean additive isotropic Gaussian noise with covariance matrix

$$
\mathbb{R}_{n n}=\sigma_{n}^{2} \mathbb{I}_{N} \in \mathcal{C}^{N \times N},
$$

where $\sigma_{n}^{2}$ denotes the noise power. However, it should be noted that the assumption of isotropic noise is not a restriction on the proposed approach provided the noise covariance can be found (up to an unknown scalar). With reference to Eq. 2 , the vector $\underline{F}_{i}(t)$ models the effect of Doppler shift due to the velocity vector of the $i$-th target $\underline{v}_{i} \in \mathcal{R}^{3 \times 1}$ and can be expressed as

$$
\begin{aligned}
\underline{F}_{i}(t) & =\exp \left(\mathrm{j} \frac{2 \pi F_{c}}{c} t\left(\rho_{i} v_{\rho_{i}} \underline{1}_{N}-\mathbf{r} \underline{v}_{i}\right) \odot \underline{\rho}_{i}^{-1}\right) \\
\text { with } \underline{\rho}_{i} & =\sqrt{\rho_{i}^{2} \cdot \underline{1}_{N}+\underline{r}_{x}^{2}+\underline{r}_{y}^{2}+\underline{r}_{z}^{2}-\frac{\rho_{i} c}{\pi F_{c}} \mathbf{r}^{T} \underline{k}\left(\theta_{i}\right)},
\end{aligned}
$$

where the parameter $t$ has been dropped from $\rho_{i}$ and $\theta_{i}$ for notational convenience and $v_{\rho_{i}}$ denotes the radial velocity of the target. The velocity vector $\underline{v}_{i}$ can be split into its radial component denoted by $v_{\rho_{i}} \underline{u}_{\rho_{\rho_{i}}} \in \mathcal{R}^{3 \times 1}$ as well as its orthoradial component denoted by $v_{\theta_{i}} \underline{u}_{v_{\theta_{i}}} \in \mathcal{R}^{3 \times 1}$ as

$$
\underline{v}_{i}=v_{\rho_{i}} \underline{u}_{\rho_{i}}+v_{\theta_{i}} \underline{u}_{v_{\theta_{i}}}
$$

noting that $\underline{u}_{v_{\theta_{i}}}$ is a unit norm vector. Note that the vector $\underline{F}_{i}(t)$ in Eq. 4 is a function of the range $\rho_{i}$ and direction $\theta_{i}$ of the emitting source with respect to the array reference point, as well as the array geometry.

With reference to Fig. 1, consider that the $N$-dimensional received signal vector $\underline{x}_{i}(t)$ is sampled with a period $T_{s}$ (point D) and passed through a bank of $N$ tapped delay lines (TDLs). Consider that each TDL is of length $N_{s}$. The output of the TDLs are concatenated and sampled with a period $N_{s} T_{s}$ to create the discretized signal vector $\underline{x}_{i}[k] \in \mathcal{C}^{N N_{s} \times 1}$ at point $\mathrm{E}$ (see Fig. 1) expressed as

$$
\underline{x}_{i}[k]=\left[\underline{x}_{1 i}^{T}[k], \underline{x}_{2 i}^{T}[k], \ldots, \underline{x}_{N i}^{T}[k]\right]^{T},
$$

where $\underline{x}_{j i}[k]=\underline{x}_{j i}\left(k T_{c s}\right) \in \mathcal{C}^{N_{s} \times 1}$ is the output from the $j$-th array element TDL. The discretized signal vector $\underline{x}_{i}[k]$ can be modelled,

\footnotetext{
${ }^{2}$ Since the array of sensors has a "large aperture", which is defined as the largest distance between any two elements in the array, the proposed model utilizes spherical wave propagation which is represented by the spherical array manifold (see Eq. 5 in [17]).
}

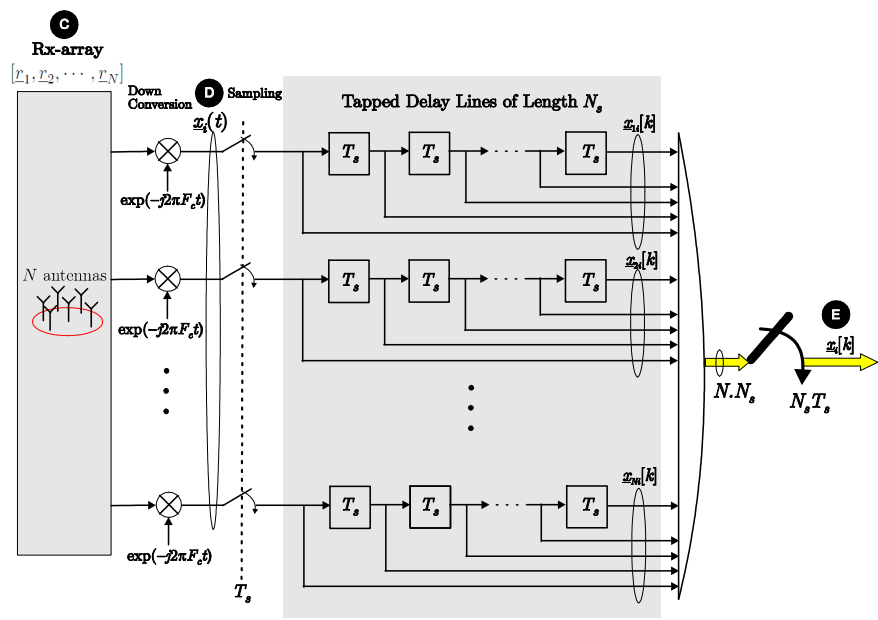

Fig. 1. Processing the received signal vector in a bank of $N$ tapped delay lines (TDLs), each of length $N_{s}$.

under the "wideband-assumption" (WB-A) as

$$
\text { WB-A: } \underline{x}_{i}[k]=\underline{\mathfrak{h}}_{i}[k] \odot\left(\underline{\mathrm{m}}_{i}[k] \otimes \underline{1}_{N_{s}}\right)+\underline{n}[k],
$$

where $\underline{\mathfrak{h}}_{i}[k] \triangleq \underline{\mathfrak{h}}\left(\rho_{i}[k], v_{\rho_{i}}[k], \theta_{i}[k], v_{\theta_{i}}[k]\right) \in \mathcal{C}^{N N_{s} \times 1}$ is defined as the Spherical SpatioTemporal ARray (Spherical STAR) manifold vector due to the $i$-th target transmission. This is a function of the array geometry (known) and the unknown time varying target parameters which are defined by the vector $\underline{z}_{i}[k] \in \mathcal{R}^{4 \times 1}$ where

$$
\underline{z}_{i}[k] \triangleq\left[\rho_{i}[k], v_{\rho_{i}}[k], \theta_{i}[k], v_{\theta_{i}}[k]\right]^{T} .
$$

For the remainder of this section, the time parameter $k$ will be dropped from $\rho_{i}, v_{\rho_{i}}, \theta_{i}$ and $v_{\theta_{i}}$, for notational convenience. Thus, in Eq. 8, the vector $\underline{\mathfrak{h}}_{i}[k]$ can be modelled as

$$
\underline{\mathfrak{h}}_{i}[k]=\left(\underline{S}_{i}[k] \otimes \underline{1}_{N_{s}}\right) \odot \underline{\mathcal{F}}_{i}[k] .
$$

Here, $\underline{S}_{i}[k]$ is assumed to change at a much slower rate than the vector $\underline{F}_{i}[k]$. In addition, $\underline{\mathcal{F}}_{i}[k] \in \mathcal{C}^{N N_{s} \times 1}$ encompasses the discretized effect of Doppler shift and is given by

$\underline{\mathcal{F}}_{i}[k]=\exp \left(\mathrm{j} \frac{2 \pi F_{c}}{c} T_{s}\left[1, \ldots, N_{s}\right]^{T} \otimes\left[\left(\rho_{i} v_{\rho_{i}} \underline{1}_{N}-\mathbf{r}^{T} \underline{v}_{i}\right) \odot \underline{\rho}_{i}^{-1}\right]\right)$,

which is a function of discretized time. Note that when the $i$-th source is in the far field of the array, the vector $\underline{\mathcal{F}}_{i}[k]$ is no longer a function of the source range, direction and the array geometry.

\section{Constant Velocity Multi-target Kinematic Model}

Consider that the $i$-th source moves with a constant velocity along a straight line relative to the array reference point. In this case, the time varying azimuth angle may be described as,

$$
\theta_{i}(t)=\theta_{i}\left(t_{0}\right)+\sin ^{-1}\left(\frac{v_{\theta_{i}}\left(t_{0}\right) \cdot T}{\rho_{i}\left(t_{0}\right)}\right)
$$

where $T$ is defined as the time elapsed since $t=t_{0}$ such that

$$
T=t-t_{0}
$$

and

$$
\rho_{i}(t)=\sqrt{\left(\rho_{i}\left(t_{0}\right)+v_{\rho_{i}}\left(t_{0}\right) T\right)^{2}+v_{\theta_{i}}^{2}\left(t_{0}\right) T^{2}} .
$$


Here, $\left(\rho_{i}\left(t_{0}\right), \theta_{i}\left(t_{0}\right)\right)$ and $\left(v_{\rho_{i}}, v_{\theta_{i}}\right)$ describe the components of the source location and velocity at $t=t_{0}$ respectively. Note that the time derivative of Eqs. 12 and 14 at $t=t_{0}$ are given by:

$$
\left.\dot{\theta}_{i}(t)\right|_{t=t_{0}}=\frac{v_{\theta_{i}}\left(t_{0}\right)}{\rho_{i}\left(t_{0}\right)},\left.\dot{\rho}_{i}(t)\right|_{t=t_{0}}=v_{\rho_{i}}\left(t_{0}\right) .
$$

Let $T$ denote the sampling time period such that $t_{0}=(k-1) T$ and $t=k T$. The unknown source parameter vector $\underline{z}_{i}[k]$ given by Eq. 9 can be used to define a discrete time state vector

$$
\underline{z}_{k i}=\left[\rho_{k i}, \dot{\rho}_{k i}, \theta_{k i}, \dot{\theta}_{k i}\right]^{T},
$$

which includes all the kinematic characteristics of the target. A suitable discrete time kinematic model for $t=k T$ is

$$
\underline{z}_{k i}=\mathbb{F}_{i} \underline{z}_{(k-1) i}+\underline{w}_{k i},
$$

where the state matrix $\mathbb{F}_{i} \in \mathcal{R}^{4 \times 4}$ is defined as

$$
\mathbb{F}_{i}=\mathbb{I}_{2} \otimes\left[\begin{array}{cc}
1, & T \\
0, & 1
\end{array}\right]
$$

and the error vector $\underline{w}_{k i}$ accounts for random perturbations about the constant velocity trajectories. These perturbations can be modelled as uncorrelated acceleration noise of zero mean with covariance matrix given by

$$
\mathbb{Q}_{i}=\left[\begin{array}{cc}
q_{\rho_{i}}^{2}, & 0 \\
0, & q_{\theta_{i}}^{2}
\end{array}\right] \otimes\left[\begin{array}{cc}
\frac{T^{3}}{3}, & \frac{T^{2}}{2} \\
\frac{T^{2}}{2}, & T
\end{array}\right],
$$

where $q_{\rho_{i}}^{2}$ and $q_{\theta_{i}}^{2}$ denote the continuous time model process noise intensity for the range-velocity and azimuth-velocity, respectively. The choice of small intensities approximates a nearly constant velocity model. Since the model is discretized, intensities have physical dimensions of length ${ }^{2} /$ time $^{3}$.

The single target discrete time kinematic model, defined in Eq. 17, can be generalised to the "multi-target" case, where $\mathrm{M}$ sources are transmitting simultaneously, as

$$
\underline{z}_{k}=\mathbb{F} \underline{z}_{k-1}+\underline{w}_{k}
$$

where

$$
\begin{aligned}
\underline{z}_{k} & =\left[\underline{z}_{k 1}^{T}, \underline{z}_{k 2}^{T}, \cdots, \underline{z}_{k M}^{T}\right]^{T} \\
\underline{w}_{k} & =\left[\underline{w}_{k 1}^{T}, \underline{w}_{k 2}^{T}, \cdots, \underline{w}_{k M}^{T}\right]^{T} \\
\mathbb{F} & =\mathbb{I}_{M} \otimes \mathbb{F}_{i} .
\end{aligned}
$$

Therefore, the covariance matrix of the discrete time state vector $\underline{z}_{k}$ defined as $\mathbb{P}_{k} \in \mathcal{R}^{4 M \times 4 M}$ is given by

$$
\mathbb{P}_{k}=\mathbb{F P}_{k-1} \mathbb{F}^{T}+\mathbb{Q},
$$

where $\mathbb{Q} \in \mathcal{R}^{4 M \times 4 M}$ is the block diagonal matrix containing all the $\mathbb{Q}_{i}$ matrices corresponding to all sources as defined in Eq. 19.

\section{Spatiotemporal Multi-Target Data Model}

Consider again that $M$ sources are transmitting simultaneously. The received signal at the output of the TDLs is now modelled as

$$
\begin{aligned}
\underline{x}[k] & =\sum_{i=1}^{M} \underline{\mathfrak{h}}_{i}[k] \odot \underline{\mathrm{m}}_{i}[k]+\underline{n}[k] \\
& =\left[\mathbb{H}[k] \odot\left(\mathbb{M}[k] \otimes \underline{1}_{N_{s}}\right)\right] \underline{1}_{M}+\underline{n}[k],
\end{aligned}
$$

where

$$
\mathbb{M}[k] \triangleq\left[\underline{\mathrm{m}}_{1}[k], \underline{\mathrm{m}}_{2}[k], \ldots, \underline{\mathrm{m}}_{M}[k]\right] \in \mathcal{C}^{N \times M},
$$

with $\underline{\mathrm{m}}_{i}[k] \in \mathcal{C}^{N \times 1}$ describing the discretized message under the WB-A and

$$
\mathbb{H}[k] \triangleq\left[\underline{\mathfrak{h}}_{1}[k], \underline{\mathfrak{h}}_{2}[k], \cdots, \underline{\mathfrak{h}}_{M}[k]\right] \in \mathcal{C}^{N N_{s} \times M},
$$

which has columns corresponding to the time varying spherical spatiotemporal manifold vectors (see Eq. 10) of the $M$ sources at the $k$-th time interval.

Equation 25 can be rewritten in a more useful and compact form as follows:

$$
\underline{x}[k]=\left(\mathbb{I}_{N N_{s}} \otimes \mathbb{H}[k]\right) \underline{\mathrm{m}}[k]+\underline{n}[k],
$$

where $\otimes$ denotes the Khatri-Rao row-wise product, which is the row-wise Kronecker product of two matrices. The vector $\underline{\mathrm{m}}[k] \in$ $\mathcal{C}^{M N N_{s} \times 1}$ denotes the vectorisation of the matrix $\left(\mathbb{M}[k] \otimes \underline{1}_{N_{s}}\right)^{T}$ formed by stacking its columns into a single column vector given by

$$
\underline{\mathrm{m}}[k]=\operatorname{vec}\left\{\left(\mathbb{M}[k] \otimes \underline{1}_{N_{s}}\right)^{T}\right\} .
$$

Therefore, in summary, based on Eqs. 20 and 28, a state space model describing the motion dynamics of multiple sources and the signals received at the output of the TDL can be defined as follows:

$$
\left.\begin{array}{l}
\underline{z}_{k}=\mathbb{F}_{k-1}+\underline{w}_{k} \\
\underline{x}_{k}=\left(\mathbb{I}_{N N_{s}} \otimes \mathbb{H}_{k}\left(\underline{z}_{k}\right)\right) \underline{m}_{k}+\underline{n}_{k}
\end{array}\right\} \mathrm{k}>0
$$

where $\underline{x}_{k} \triangleq \underline{x}[k], \underline{n}_{k} \triangleq \underline{n}[k], \mathbb{H}_{k}\left(\underline{z}_{k}\right)=\mathbb{H}[k]$ and $\underline{m}_{k}=\underline{\mathrm{m}}[k]$. This is the standard structure of a discrete time state space model which, however, involves the motion parameterisation described before and also the Spherical STAR manifold vectors $\underline{\mathfrak{h}}_{i}[k], i=1,2, \ldots, M$, associated with the $M$ sources, taking into account the "widebandassumption". With reference to Eq. 30, the process noise $\underline{w}_{k} \in$ $\mathcal{R}^{4 M \times 1}$ associated with the state space model and the measurement noise $\underline{n}_{k} \in \mathcal{C}^{N N_{s} \times 1}$ associated with the wireless channel are assumed to have zero mean and known covariance matrices $\mathbb{Q}$ and $\sigma_{n}^{2} \mathbb{I}_{N N_{s}}$, respectively. Furthermore, the initial state vector $\underline{z}_{0}$ has a mean of $\mathcal{E}\left\{\underline{z}_{0}\right\}$ and a covariance defined by the matrix $\mathbb{P}_{0}$. The first and second order statistics of $\left\{\underline{z}_{0}, \underline{w}_{k}, \underline{n}_{k}\right\}$ can be compactly restated as follows:

$$
\begin{aligned}
& \mathcal{E}\left\{\left[\underline{w}_{k}^{T}, \underline{n}_{k}^{T}, \underline{z}_{0}^{T}, 1\right]^{T}\left[\underline{w}_{m}^{H}, \underline{n}_{m}^{H}, \underline{z}_{0}^{H}\right]\right\}= \\
& {\left[\begin{array}{ccc}
{\left[\begin{array}{ll}
\mathbb{Q}, & \mathbb{O}_{4 M \times N N_{s}} \\
\mathbb{O}_{N N_{s} \times 4 M}, & \sigma_{n}^{2} \mathbb{I}_{N N_{s}}
\end{array}\right] \delta_{k-m},} & \mathbb{O}_{4 M \times 4 M} \\
\mathbb{O}_{4 M \times 4 M}, & \mathbb{O}_{4 M \times N N_{s}} & \mathbb{O}_{N N_{s} \times 4 M} \\
\underline{0}_{4 M}^{T}, & \underline{0}_{N N_{s}}^{T} & \mathcal{E}\left\{\underline{z}_{0}\right\}^{H}
\end{array}\right]}
\end{aligned}
$$

where $\mathbb{O}_{p \times q}$ denotes a $(p \times q)$ matrix of zeros.

\section{Performance Evaluation}

The simulation environment for utilising Eq. 30 is described as follows. The array used for tracking is defined as

$$
\mathbf{r}=\left[\begin{array}{ccccccc}
0, & 50, & 30, & -30, & 30, & 10, & -50 \\
0, & 0, & 45, & -50, & -62, & 75, & 29 \\
0, & 0, & 0, & 0, & 0, & 0, & 0
\end{array}\right]
$$




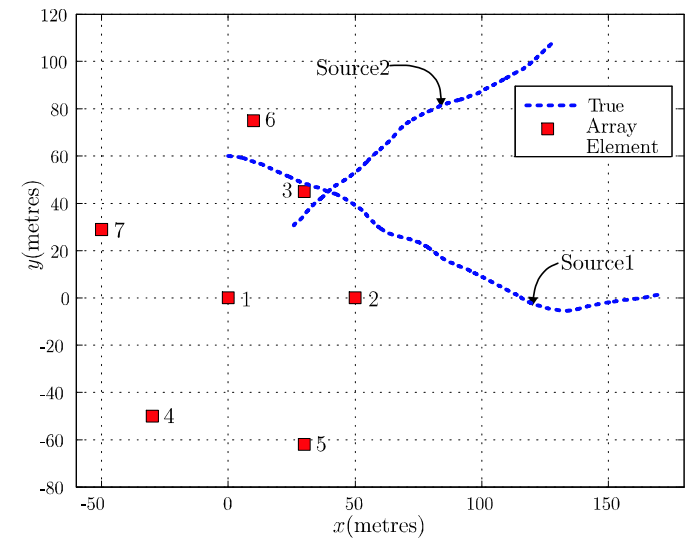

Fig. 2. Position Tracking: True trajectories of two sources to be tracked using the $N=7$ element array in Eq. 32 .

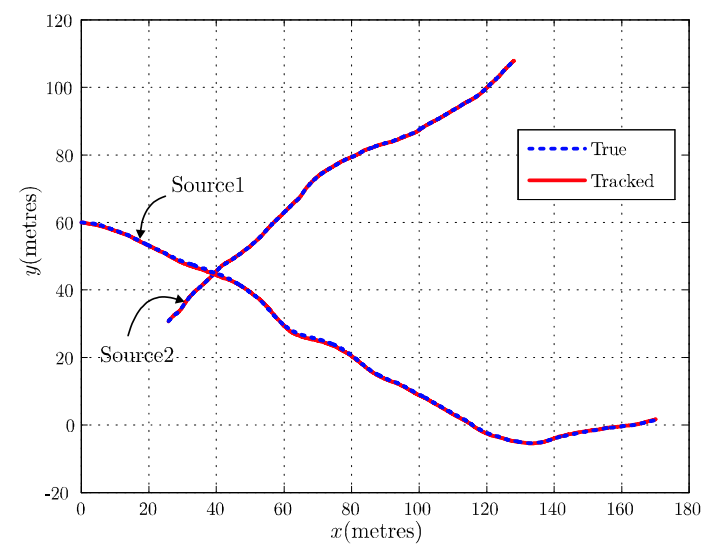

Fig. 3. Position Tracking: True trajectories and tracked positions for the environment of Fig. 2 with $N_{s}=10$ and $\mathrm{SNR}_{\text {in }}=0 \mathrm{~dB}$.

measured in meters. The chosen array does not possess any special geometrical structure. Two sources of equal power are tracked using the array of Eq. 32. The signals of the sources are simulated as independent identically distributed Gaussian processes with a unity variance and the carrier frequency $F_{c}$ is assumed to be $2.4 \mathrm{GHz}$. The targets are moving in the same plane as the sensor array. The array elements and the trajectories of the sources in the $x-y$ plane are shown (blue dotted lines) in Fig. 2.

Tracking is assumed to be performed, using an extended Kalman filter, over an interval of $5 \mathrm{~s}$ with $T=1 \mathrm{~ms}$. Hence, there are a total of 5000 snapshots of data available at the end of the tracking interval. The initial locations of the targets are assumed to be known within 1 meter (e.g. estimated using any high resolution localization approach as in [17]) with initial covariance matrix $\mathbb{P}_{0}=\mathbb{I}_{4 M \times 4 M}$. The selected level of the range process noise is $q_{\rho_{i}}^{2}=30 \mathrm{~m}^{2} / \mathrm{sec}^{3}$ and the direction (angle) process noise is $q_{\theta_{i}}^{2}=0.035 \mathrm{rad}^{2} / \mathrm{sec}^{3}$, where $i=1,2$. Note that it is impractical to assume full knowledge of the true process noise statistics (covariance matrix). However, any "uncertainty" has no significant effect in the tracking performance.

For the purposes of simulation, the length of TDL for each element

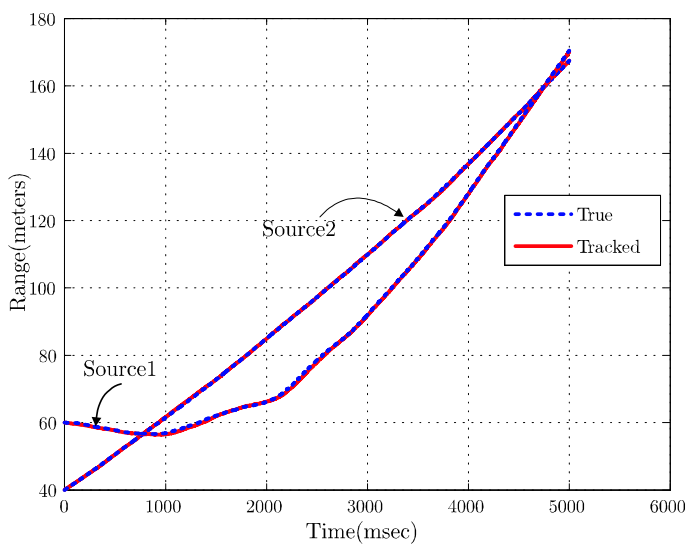

Fig. 4. Range Tracking: True and tracked ranges for the environment of Fig. 2 with $N_{s}=10$ and $\mathrm{SNR}_{\text {in }}=0 \mathrm{~dB}$.

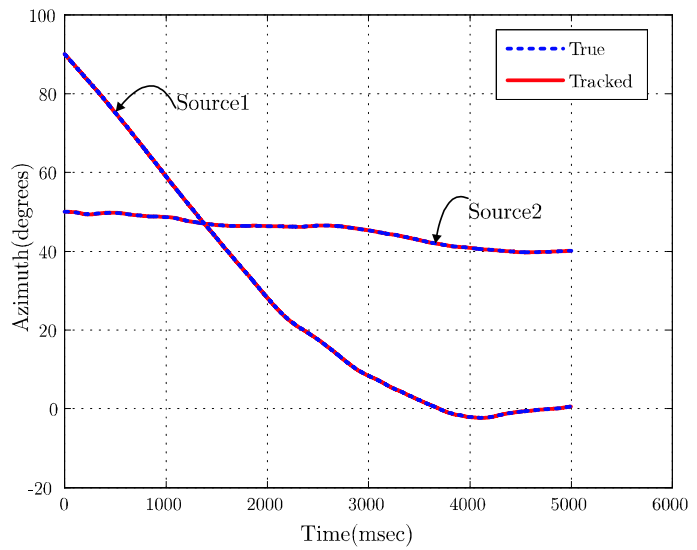

Fig. 5. Azimuth Tracking: True and tracked azimuth angles for the environment of Fig. 2 with $N_{s}=10$ and $\mathrm{SNR}_{\text {in }}=0 \mathrm{~dB}$.

is assumed to be 10 (i.e. $N_{s}=10$ ). The array data is simulated for $\mathrm{SNR}_{\text {in }}=0 \mathrm{~dB}$. Figure 2 provides a plot of the two target true trajectories. Figures 3 to 5 provide a plot of the true target motion parameters in dotted blue lines superimposed by red solid lines representing the corresponding estimated parameters in a typical sample run. From Figs. 3, 4 and 5, it is clear that the trajectories of the 2 targets are accurately estimated for $\mathrm{SNR}_{\text {in }}=0 \mathrm{~dB}$.

\section{CONCLUSIONS}

In this paper, the problem of tracking multiple moving targets was addressed using large aperture arrays. In particular, a novel sphericalspatiotemporal-state-space model was presented incorporating the array geometry in conjunction with time varying functions of the ranges, directions and Doppler effects of the targets. An example of simulation results was presented to demonstrate the proposed model in tracking the locations and velocities of the targets. Some applications of this model include battlefield communications, where mobile soldiers may form the wide aperture sensor array, and cellular communications, where locating mobile sources/targets enables high precision beamforming to be deployed. 


\section{REFERENCES}

[1] A. Roy, S. Das, and A. Misra, "Exploiting information theory for adaptive mobility and resource management in future cellular networks," IEEE Wireless Communications, vol. 11, no. 4, pp. 59 - 65, Aug. 2004.

[2] A. Farina and S. Pardini, "Survey of radar data-processing techniques in air-traffic-control and surveillance systems," IEE Proceedings F Communications, Radar and Signal Processing, vol. 127, no. 3, pp. 190 -204, Jun. 1980.

[3] R. Kenefic and P. Goulette, "Sensor netting via the discrete time extended Kalman filter," IEEE Transactions on Aerospace and Electronic Systems, vol. AES-17, no. 4, pp. $482-489$, Jul. 1981.

[4] D. Balakrishnan and A. Nayak, "An efficient approach for mobile asset tracking using contexts," IEEE Transactions on Parallel and Distributed Systems, vol. 23, no. 2, pp. 211 -218, Feb. 2012.

[5] S. Tao, V. Manolopoulos, S. Rodriguez, M. Ismail, and A. Rusu, "Hybrid vehicle positioning and tracking using mobile phones," in 11th International Conference on Telecommunications (ITST), Aug. 2011, pp. $315-320$.

[6] A. Oka and L. Lampe, "Distributed target tracking using signal strength measurements by a wireless sensor network," IEEE Journal on Selected Areas in Communications, vol. 28, no. 7, pp. 1006 -1015, Sep. 2010.

[7] W.-L. Yeow, C.-K. Tham, and W.-C. Wong, "Energy efficient multiple target tracking in wireless sensor networks," IEEE Transactions on Vehicular Technology, vol. 56, no. 2, pp. 918 -928, Mar. 2007.

[8] Y. Zhou, P. C. Yip, and H. Leung, "Tracking the direction-of-arrival of multiple moving targets by passive arrays : Algorithm," IEEE Transactions on Signal Processing, vol. 47, no. 10, pp. 2655-2666, Oct. 1999.

[9] V. Cevher and J. McClellan, "General direction-of-arrival tracking with acoustic nodes," IEEE Transactions on Signal Processing, vol. 53, no. 1, pp. 1-12, Jan. 2005.
[10] M. Orton and W. Fitzgerald, "A Bayesian approach to tracking multiple targets using sensor arrays and particle filters," IEEE Transactions on Signal Processing, vol. 50, no. 2, pp. 216-223, Feb. 2002.

[11] P. Chavali and A. Nehorai, "Scheduling and power allocation in a cognitive radar network for multiple-target tracking," IEEE Transactions on Signal Processing, vol. 60, no. 2, pp. 715-729, Feb. 2012.

[12] R. Niu and R. Blum, "Target localization and tracking in noncoherent multiple-input multiple-output radar systems," IEEE Transactions on Aerospace and Electronic Systems, vol. 48, no. 2, pp. 1466-1489, Apr. 2012.

[13] D. Henke, C. Magnard, M. Frioud, D. Small, E. Meier, and M. Schaepman, "Moving-target tracking in single-channel wide-beam SAR," IEEE Transactions on Geoscience and Remote Sensing, vol. 50, no. 11, pp. 4735-4747, Nov 2012.

[14] J. Hu, X. li Ding, Z. wei Li, J. jun Zhu, Q. Sun, and L. Zhang, "Kalmanfilter-based approach for multisensor, multitrack, and multitemporal InSAR," IEEE Transactions on Geoscience and Remote Sensing, vol. 51, no. 7, pp. 4226-4239, Jul. 2013.

[15] K. Luo and A. Manikas, "Superresolution multitarget parameter estimation in MIMO radar," IEEE Transactions on Geoscience and Remote Sensing, vol. 51, no. 6, pp. 3683-3693, Jun. 2013.

[16] A. Haimovich, R. Blum, and L. Cimini, "MIMO radar with widely separated antennas," IEEE Signal Processing Magazine, vol. 25, no. 1, pp. 116-129, Dec. 2008.

[17] A. Manikas, Y. Kamil, and M. Willerton, "Source localization using large aperture sparse array," IEEE Transactions on Signal Processing, vol. 60 , no. 12 , pp. 1-13, Dec. 2012. 